\title{
ARTICLE
}

\section{The effect of 'Sensor Lock', a knee-ankle-foot orthosis with an electromechanical stance control knee joint, on walking parameters and gait symmetry of subjects with quadriceps weakness: a pilot study}

\author{
Farnoosh Asadi ${ }^{1,2,3}$, Mokhtar Arazpour ${ }^{1,2,4}$, Monireh Ahmadi Bani ${ }^{2}$, Gholamreza Aminian ${ }^{2}$ and Reza Vahab Kashani $^{2}$
}

STUDY DESIGN: Pilot study.

OBJECTIVES: This study aimed to evaluate an electromechanical stance control (SC) knee joint, 'sensor lock', which could potentially solve the problems of walking parameters and gait symmetry in subjects with poliomyelitis.

SETTING: University of Social Welfare and Rehabilitation Sciences.

METHODS: Six subjects with quadriceps weakness were enrolled in this study. A custom-made stance-control knee-ankle-foot orthosis (SCKAFO) with the same set of components was constructed for each participant. A motion analysis system was used for analysis of lower limb kinematics.

RESULTS: There were no significant differences between the knee-ankle-foot orthoses (KAFOs) and the two types of knee joints with regard to temporal-spatial parameters. Walking speed and cadence reduced and stride length increased in the stance control (SC) mode compared to the knee joint locked mode. The maximum knee flexion angle during swing phase increased when walking with SC mode compared to walking with the KAFO in knee joint lock mode. There was a significant difference between two test conditions with regard to hip flexion. There were only significant differences in the symmetry index of the knee flexion between two test conditions.

CONCLUSION: Compared to locked knee mode, SC mode demonstrated a slower speed of walking and increased peak knee flexion during swing. Regarding gait symmetry, the symmetry index of the knee flexion and speed of walking decreased when the KAFO with SC mode was used compared to KAFO with knee joint locked mode.

Spinal Cord Series and Cases (2017) 3, 17035; doi:10.1038/scsandc.2017.35; published online 15 June 2017

\section{INTRODUCTION}

People with lower limb weakness, especially in the quadriceps muscles usually use knee-ankle-foot orthoses (KAFOs) for standing and walking. Quadriceps weakness is seen in patients with polio, spine bifida, multiple sclerosis, stroke, and incomplete spinal cord injury. ${ }^{1}$

Different types of the knee joint, including knee joint locked in extension position, stance control knee joint, and powered knee joint have been used in KAFOs. Knee joint locked provides a knee joint locked during walking and limits knee joint flexion in ambulation. This feature can cause an abnormal gait pattern such as hip hiking, leg circumduction, vaulting, pain, loss of motion in lower back, and greater energy consumption. These patients also suffer from cosmetic problems in ambulation. ${ }^{2-5}$ Problems with KAFOs include weight, cosmetics, fit and comfort and difficulty in donning and doffing lead to rejection of KAFOs. ${ }^{1}$ Studies have shown that over $40 \%$ of KAFO users are dissatisfied with their orthoses and eventually between 58 and $79 \%$ of KAFO users stop using their orthoses and return to wheelchair. ${ }^{3,4}$ More over chronic use of wheelchair can cause problems such as neuropathy, painful shoulder, contracture in hip and knee, carpal tunnel syndrome and osteoarthritis. ${ }^{5}$

Stance-control KAFO (SCKAFO) is a new generation of the orthosis designed for subjects with quadriceps weakness. This type of orthosis provides free knee flexion during swing but prevents knee flexion during stance phase of gait. ${ }^{6}$ There are different types of the stance-control (SC) knee joint. The Otto Bock Free Walk/Becker, Horton's Stance Control Orthosis, Filature Swing Phase Lock, Becker Orthopedic 9001 E-Knee, Ottawalk Belt-Clamping Knee Joint, Dynamic Knee Brace System, and the Dual Stiffness Knee Joint are all examples. High cost, large size, excessive weight, and poor function are limitations of the current SC knee joints; ${ }^{1}$ therefore, few SCKAFOs are clinically and commercially suitable. Although improvements in ambulation and walking ability with SCKAFOs, compared to fixed-knee KAFOs, were reported in previous studies, ${ }^{1,6-10}$ some SC knee joints designs are bulky and heavy. These factors may influence energy consumption, orthosis use, and walking. Size, weight, and noise are factors that affect orthosis acceptance in KAFO users. Cosmetically, the KAFO must have knee joint components with

\footnotetext{
${ }^{1}$ Pediatric Neurorehabilitation Research Center, University of Social Welfare and Rehabilitation Sciences, Tehran, Iran; ${ }^{2}$ Orthotics and Prosthetics Department, University of Social Welfare and Rehabilitation Sciences, Tehran, Iran; ${ }^{3}$ Student research commute, University of Social Welfare and Rehabilitation Sciences, Tehran, Iran and ${ }^{4}$ Iranian Research Center on Aging, University of Social Welfare and Rehabilitation Sciences, Tehran, Iran.

Correspondence: M Arazpour (M.arazpour@yahoo.com)

Received 22 February 2017; revised 14 May 2017; accepted 17 May 2017
} 
Table 1. Subjects demographic information

\begin{tabular}{|c|c|c|c|c|c|c|c|c|c|c|c|c|c|}
\hline \multirow[t]{2}{*}{ Subject } & \multirow{2}{*}{$\begin{array}{c}\text { Weight, } \\
\text { kg }\end{array}$} & \multirow{2}{*}{$\begin{array}{l}\text { Affected } \\
\text { side }\end{array}$} & \multirow[t]{2}{*}{ Gender } & \multirow{2}{*}{$\begin{array}{l}\text { Limb length } \\
\text { discre pancy, } \\
\mathrm{cm}\end{array}$} & \multirow{2}{*}{$\begin{array}{l}\text { Height, } \\
\mathrm{cm}\end{array}$} & \multirow{2}{*}{$\begin{array}{c}\text { Age, } \\
y\end{array}$} & \multirow{2}{*}{$\begin{array}{c}B M l, \\
\mathrm{~kg} \mathrm{~m}^{-2}\end{array}$} & \multicolumn{6}{|c|}{ Muscle strength } \\
\hline & & & & & & & & $\begin{array}{l}\text { Hip } \\
\text { flexion }\end{array}$ & $\begin{array}{c}\text { Hip } \\
\text { extension }\end{array}$ & $\begin{array}{l}\text { Knee } \\
\text { flexion }\end{array}$ & $\begin{array}{c}\text { Knee } \\
\text { extension }\end{array}$ & $\begin{array}{c}\text { Ankle } \\
\text { dorsi } \\
\text { flexion }\end{array}$ & $\begin{array}{l}\text { Ankle } \\
\text { plantar } \\
\text { flexion }\end{array}$ \\
\hline 1 & 65 & Right & Female & 3.5 & 160 & 53 & 25.39 & $4-$ & 3 & $4-$ & $3-$ & 4 & 3 \\
\hline 2 & 58 & Right & Female & 1 & 157 & 48 & 23.57 & 4 & $4-$ & 3 & 3 & 4 & 3 \\
\hline 3 & 82 & Left & Male & 7 & 175 & 47 & 26.79 & 4 & $4+$ & $3+$ & 3 & 4 & 3 \\
\hline 4 & 70 & Left & Male & 7 & 174 & 37 & 23.17 & $4-$ & $4-$ & $4-$ & $3-$ & $4-$ & 4 \\
\hline 5 & 74 & Right & Male & - & 168 & 57 & 26.24 & 4 & $4+$ & $3-$ & 3 & 4 & $4-$ \\
\hline 6 & 74 & Right & Female & 3.5 & 158 & 43 & 29.71 & 4 & 3 & 3 & 3 & $3+$ & 3 \\
\hline $\begin{array}{l}\text { Mean } \\
\text { (s.d.) }\end{array}$ & $\begin{array}{l}70.5 \\
(8.2)\end{array}$ & - & - & - & $\begin{array}{c}165.33 \\
(8.09)\end{array}$ & $\begin{array}{c}47.5 \\
(7.09)\end{array}$ & $\begin{array}{l}25.81 \\
(3.19)\end{array}$ & & & & & & \\
\hline
\end{tabular}

Abbreviation: BMI, body mass index.

smaller dimensions in mediolateral and anteroposterior directions, which may reduce orthosis weight. Recently a new kind of SC knee joint has been developed in the University of Social Welfare and Rehabilitation Sciences called the 'sensor lock.' This design is similar to the Horton device, but with a 'magnetic switch' that engages and disengages a pawl section. The 'sensor lock' had $60 \times 90 \times 12 \mathrm{~mm}$ dimensions.

Patients with polio compared to able-bodied subjects have weight bearing on the non-paretic limb and therefore gait asymmetry. A reduced stance phase percentage time and reduced step length on the affected side, plus reduced walking speed have been reported in walking of patients with poliomyelitis. ${ }^{11}$ Since a symmetrical gait pattern is important for increasing independent mobility in this kind of subjects, evaluation of this parameter, gait symmetry, is important in these patients. Therefore this pilot study aimed to evaluate an electromechanical SC knee joint -'sensor lock'- which could potentially solve problems related to walking parameters and gait symmetry in patients with poliomyelitis.

\section{MATERIALS AND METHODS}

\section{Participants}

Six patients with quadriceps weakness (body mass index: 20.7-29.71 $\mathrm{kg} \mathrm{m}^{-2}$, weight: $56-82 \mathrm{~kg}$, age: $46.71 \pm 6.29$ years, age range: $37-53$ years) were enrolled in this study. Table 1 presents the demographic information of subjects in this study.

Inclusion criteria were included subjects who routinely used KAFOs unilaterally with lockable orthotic knee joints, ability to walk a minimum of $50 \mathrm{~m}$, and with sufficient hip flexor strength (at a minimum level equivalent to level 3 of the Oxford scale) to advance the limb during swing phase. Exclusion criteria included the existence of impaired cognition, poor balance and soft tissue contractures greater than $15^{\circ}$ at the hip, $10^{\circ}$ at the knee or $5^{\circ}$ at the ankle, orthostatic hypotension, cardiac arrhythmias, vestibular dysfunction and peripheral neuropathy, which could contribute to falling, or having near falls, or loss of balance. ${ }^{12}$ The study was approved by the Ethics Committee of University of Social Welfare and Rehabilitation Sciences. All subjects signed informed consent prior to participation in the study.

\section{Interventions}

According to what was discussed, a custom-made KAFO was fabricated by an experienced orthotist for each subject (Figure 1). The KAFOs had molded thigh cuffs and solid ankle foot orthoses. The exact location of knee and ankle joints, longitudinal distance between the knee and ankle joints, diameter of ankle and knee joints, medial longitudinal distance between perineum to the knee joint associated with precision casting of the lower limbs were considered as important parameters in the construction of KAFO for each patient.
SC knee joint. The new design contains a foot switch with rubber bed under the heel, a magnetic switch, and a 1-way lock mechanism that includes a 6-teeth pawl (Figure 1). The sensor lock mechanism is based on the SC concept, that is, the knee joint gets locked in the stance phase and free knee motion is provided in the swing phase.

Control of SC knee joint. In the stance phase, the spring force pushes the pawl up against gravity and engages it with the ratchet. At the end of preswing, the heel sensor is off loaded and the magnetic switch activates and allows the knee flexion for swing. The solenoid is stimulated in the swing phase and the knee joint moves freely in both directions. In the next initial contact, the actuator is deactivated and the spring force pushes up the pawl and locks the knee only in flexion direction to avoid falling risk but extension is permitted.

Battery. A rechargeable $24 \mathrm{~V}$ battery with $2 \mathrm{~h}$ of available continuous working was used to provide power for the actuator. When the battery gets low or removed from the circuit for any reason, the SCKAFO functions similar to a traditional KAFO.

Gait training with SCKAFO. After preparation of orthoses for subjects, a supervised gait training program was performed in a rehabilitation center under controlled and supervised conditions for all subjects. The main goal of the gait training was to achieve confidence and experience in balance, standing, and walking with the orthosis.

The orthotic gait training was performed on the level ground $2 \mathrm{~h}$ per day, 3 day per week and for 2 week. This program included five phases. Phase one was performed in the pre-orthosis wearing to increase strength of the pelvic and abdominal muscles to provide perception of pelvic rotation and retroversion. Phase 2 was to permit the KAFO user to get self dependence via standing and weight bearing with the SCKAFO with the locked knee joint position within the parallel bars. Phase 3 composed walking in the SCKAFO with emphasis on the stance phase. The main goal of this stage was to perform locking and unlocking of the stance control knee joint during walking. Locking of the stance control knee joint in the stance phase from initial contact to terminal stance was important in this stage to provide stability. In addition, equality of the step length in this phase educated during training. Phase 4 was walking with SCKAFO with emphasis on the swing phase. Unlocking of the stance control knee joint at terminal stance and generation of the extensor momentum to provide extension of the knee joint before next step were trained in this stage. Walking with SCKAFO outside the parallel bars was considered as the final stage of the orthotic gait training.

\section{Data collection}

A camera system (Vicon, 460, Oxford Metrics, Inc., Oxford, UK) at a frequency of $100 \mathrm{~Hz}$ was used for capturing subjects' motion during walking with the orthosis. Passive reflective markers were placed bilaterally over the jugular notch, the spinous process of the seventh cervical vertebrae, bilaterally over the acromioclavicular joints, the anterior superior iliac spine, the greater trochanter, the lateral condyle of the femur, the head and lateral malleolus of the fibula, the calcaneus and on the dorsum 
of the foot over the second metatarsal head. Reflective markers were placed on the orthosis (affected side) and the skin (non-affected side) of the subjects. All subjects walked three times at their self-selected comfortable speed along a 6-m long marked walkway in a gait analysis laboratory. Walking analysis was randomly performed in two conditions, stance control mode and locked knee joint mode. Subjects had $\sim 20 \mathrm{~min}$ accommodation time before walking analysis with the orthosis. Data were processed at $100 \mathrm{~Hz}$ with VICON Body Builder (Oxford Metrics, Inc.) using the standard lower limb model included in the software. The data were then analyzed using MATLAB (Math Works, Natick, MA, USA).

The gait symmetry index (SI) for the stride length and speed was calculated according to the following equation: ${ }^{13}$

$$
\text { SI }(\%)=\frac{(\mathrm{XL}-\mathrm{XR})}{1 / 2(\mathrm{XL}+\mathrm{XR})} * 100
$$

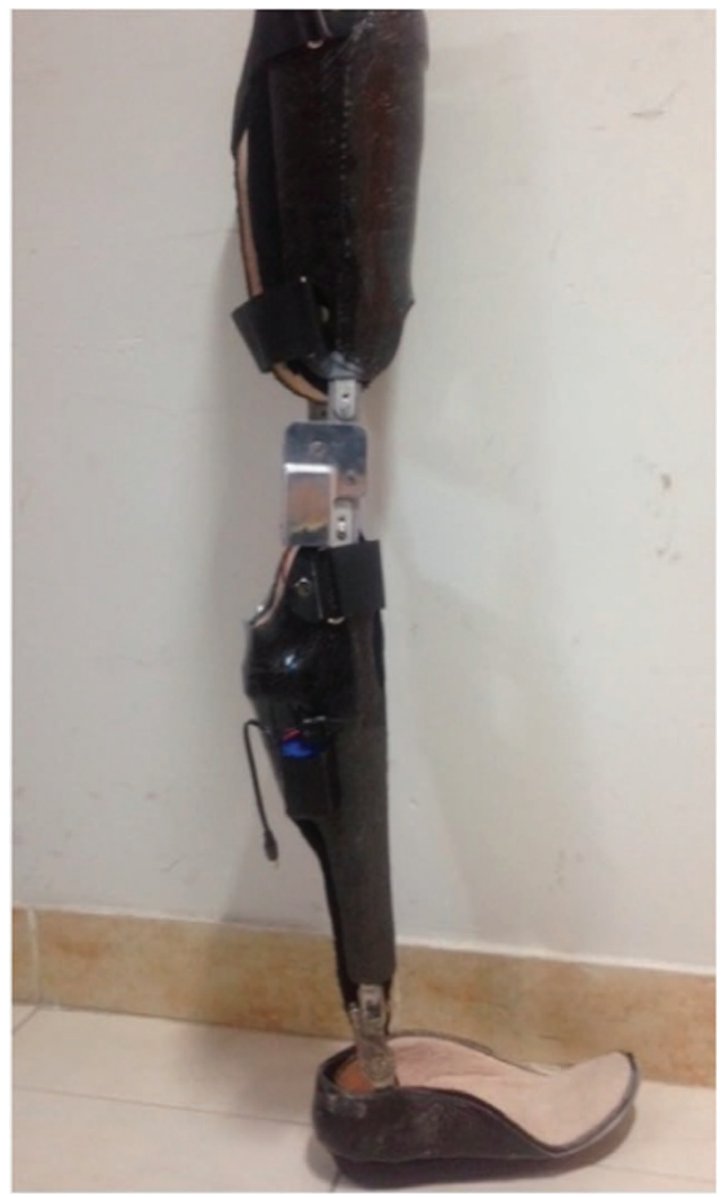

Figure 1. KAFO used in this study. where $\times L$ and $\times R$ are the means of a spatial or temporal parameter of the left and right leg, respectively. A SI value of zero represents complete symmetry. The degree of asymmetry could range from -200 to $+200 \%$ as the difference between the two sides is reported against their average value. The primary outcome measure were walking speed $\left(\mathrm{m} \mathrm{s}^{-1}\right)$, stride length $(\mathrm{m})$, cadence (steps per min), max knee flexion in swing (deg), hip joint flexion and extension (deg). Secondary outcome measures included gait symmetry.

\section{Statistical analysis}

Normality was confirmed by the Kolmogorov-Smirnov technique. A Wilcoxon signed-rank test $(P<0.05)$ was used to compare two test conditions. JMP IN statistical software (SAS Institute, Inc., Cary, NC, USA) was used for data analysis.

\section{RESULTS}

Temporal-spatial parameters

Table 2 presents the mean spatiotemporal parameters of the participants using the KAFO in two different modes (stance control and the knee lock in full extension).

Walking speed was reduced in the stance control mode compared to the locked knee mode position (SC mode; $0.31 \mathrm{~m} \mathrm{~s}^{-1}$ and locked in full knee extension; $0.32 \mathrm{~m} \mathrm{~s}^{-1}$ ), but in the affected side, walking with KAFO in the SC mode also provided a longer stride length compared to walking with KAFO in the locked knee mode (SC mode; $0.68 \mathrm{~m}$ and locked in full knee extension mode; $0.65 \mathrm{~m}$ ). Cadence was reduced in the SC mode compared to the locked knee mode (Table 2). There were no significant differences between KAFOs with two types of knee joints in this study with regard to temporal-spatial parameters (Table 2).

\section{Kinematics}

The maximum knee flexion angle during swing phase increased when walking with SC mode compared to walking with the KAFO in knee joint lock mode (SC mode: $25^{\circ}$, locked mode: $4^{\circ}$ ). The maximum hip joint increased and maximum hip extension decreased when walking with SC mode compared to walking with the KAFO in knee joint lock mode (Table 2). These differences were all statistically significant (maximum knee flexion angle during swing phase, $P$-value: 0.009 , hip flexion, $P$-value: 0.03 ).

\section{Gait symmetry index}

Table 3 presents symmetry index (SI) values of the two walking conditions in this study. There were no significant differences in the $\mathrm{SI}$ of step length or speed of walking between walking with KAFO with SC mode and locked knee joint mode. A different speed was calculated for each leg. Using the KAFO with SC mode knee joint significantly decreased the gait SI when applied to knee flexion during swing phase $(P=0.009)$ compared to walking with the KAFO with dropped locked knee joint mode.

Table 2. Mean (s.d.) values of mentioned parameters on the affected and unaffected sides in walking with two different types of KAFO (lock knee and SC mode)

\begin{tabular}{lcc}
\hline & & Walking condition \\
\cline { 2 - 3 } & Lock knee mode, mean \pm s.d. & SC mode, mean \pm s.d. \\
\hline Walking velocity $\left(\mathrm{m} \mathrm{s}^{-1}\right.$ ) & $0.32 \pm 0.08$ & $0.31 \pm 0.10$ \\
Cadence (steps/min) & $37.1 \pm 10.1$ & $35.27 \pm 14.54$ \\
Stride length (m) & $0.65 \pm 0.1$ & $0.68 \pm 0.15$ \\
Maximum knee flexion in swing phase (degrees) & $4.64 \pm 1.54$ & 0.737 \\
Maximum hip flexion (degrees) & $30.56 \pm 13.34$ & 0.205 \\
Maximum hip extension (degrees) & $8.73 \pm 3.19$ & $39.18 \pm 17.78$ \\
Abbreviations: KAFO, knee-ankle-foot orthoses; SC, stance control. & & $7.93 \pm 0.67$ \\
\hline
\end{tabular}


Table 3. The mean, s.d. and comparison of the SI of various parameters in the two walking test conditions

\begin{tabular}{|c|c|c|c|c|c|c|}
\hline & SC & Lock & SC & Lock & SC & Lock \\
\hline
\end{tabular}

\section{DISCUSSION}

In this pilot study we analyzed objective biomechanical tests to investigate walking parameters between two different types of knee joint (locked in extension and SC mode) for KAFOs. Providing knee flexion in swing phase with stability in stance is the main goals of SC knee joints. Knee flexion in swing phase for ambulation was provided with the KAFO in SC mode at a mean value of $25^{\circ}$. Providing knee flexion in swing phase is important and critical to provide foot clearance and reduces compensatory motions such as circumduction, vaulting gait and hip hiking during walking. Provided knee flexion in swing phase in this pilot study was $25 \pm 17^{\circ}$. The mean value of this parameter had a reduced rate compared to normal walking. Compared to previous studies in this field, the mean value of this parameter was reported $44^{\circ}$ when wearing KAFO with SC mode compared to the KAFO with dropped locked knee joint. ${ }^{6,7,14}$ The mean value of this parameter had a reduced rate compared to values found in other studies in this field. This aspect suggests that patients newly fitted with KAFO with SC knee joints should have proper gait training with this type of orthosis.

Subjects with lower limb weakness need gait training to adapt to new orthoses (20). Previous studies evaluating the use of KAFOs with SC mode reported different training times. In our study, mean walking speed did not significantly increase when walking with the SCKAFO compared to KAFO with locked knee joint mode after gait training. In contrast Irby et al. ${ }^{7}$ provided 6 months of orthotic gait training with KAFO with SC knee joint and noted improvement in walking speed. It is likely we did not provide sufficient gait training in our study.

In addition our study participants were experienced KAFO users who used orthoses with locked knee position and this may have made it more difficult to use the new system. ${ }^{7}$ In other studies, increased speed and cadence were reported in using the automatic mode in a SCKAFO compared to wearing the locked mode KAFO. ${ }^{7,8,15}$ However other reports showed, no change between the locked and auto modes in terms of temporospatial parameters. ${ }^{6,16}$ Longer times of accommodation with the orthosis and more experience in using KAFO with SC knee joint is therefore required to enable subjects to produce faster walking speeds when walking with this type of orthosis.

Compared to normal gait parameters, post polio syndrome patients showed inter-subject variability in most of the spatiotemporal parameters and SI showed greater and significant differences. ${ }^{11}$ Subjects with poliomyelitis had slower speed of walking, decreased cadence, prolonged stance duration, and shorter step lengths. ${ }^{11}$ The SI of step length increased. The SI of the knee flexion and speed of walking decreased when the KAFO with SC mode was used compared to KAFO with lock knee joint mode. Based on the improvement of the swing phase flexion illustrated with the SC knee joint in wearing KAFO, improvement of gait symmetry during swing may be expected.

From clinical view, to provide lower limb stability in ambulation in subjects with quadriceps weakness, traditional KAFO's lock the knee joint in full extension, but eliminating knee flexion forces individuals to make compensations and to ambulate with gait deviations. SCKAFO's supported the affected lower extremity during weight bearing via resisting knee flexion during stance phase; but permit free knee motion during swing phase therefore facilitate moving forward with minimal compensatory motions. Lower extremity muscle weaknesses and strength deficits, limitation of the range of motion and the functional biomechanical shortcoming were considered to choose the orthotic knee joint options in KAFOs.

Our study is limited by the low sample size, short time of gait training with SC knee joint, evaluation of the KAFO with SC knee joint by experienced locked knee KAFO users instead of new or experienced SCKAFO users, and no analysis of the KAFO influence with SC mode on energy consumption. Further studies which resolve these limitations would be beneficial. Ankle angles (vaulting) or hip rotation (circumduction) and patient's satisfaction are also important to assess. In addition, our study included three males and three females and evaluation of the gender differences on walking parameters and gait symmetry were not considered in this study and this could be investigated in the future.

\section{CONCLUSION}

This study evaluated the effects of a KAFO with two different knee joint actions (SC mode and locked knee joint) on gait parameters of subjects with quadriceps weaknesses. The KAFO with SC mode provided knee flexion in swing phase associated with stability in stance as compared to use of KAFOs with locked knee joints. Compared to the locked knee mode, using SC mode demonstrated a slower speed of walking and increased peak knee flexion during swing in this study. Evaluation of this knee joint with long-time gait training will be beneficial in this field. With regard to gait symmetry, the $\mathrm{SI}$ of the knee flexion and speed of walking decreased by wearing KAFO with SC mode compared to KAFO with lock knee joint mode.

\section{ACKNOWLEDGEMENTS}

We thank the iranian National Science Foundation for financial support of this study.

\section{AUTHOR CONTRIBUTIONS}

All authors equally contributed in the preparation of this manuscript.

\section{COMPETING INTERESTS}

The authors declare no conflict of interest.

\section{REFERENCES}

1 Yakimovich T, Kofman J, Lemaire ED. Design and evaluation of a stance-control knee-ankle- foot orthosis knee joint. IEEE Trans Neural Syst Rehabil Eng 2006; 14: 361-369.

2 Bernhardt K, Oh T, Kaufman K. Stance control orthosis trial in patients with inclusion body myositis. Prosthet Orthot Int 2011; 35: 39-44. 
3 Hwang S, Kang S, Cho K, Kim Y. Biomechanical effect of electromechanical knee-ankle-foot-orthosis on knee joint control in patients with poliomyelitis. Med Biol Eng Comput 2008; 46: 541-549.

4 Rasmussen AA, Smith KM, Damiano DL. Biomechanical evaluation of the combination of bilateral stance-control knee-ankle-foot orthoses and a reciprocating gait orthosis in an adult with a spinal cord injury. J Prosthet Orthot 2007; 19: 42-47.

5 Sutherland D, Santi M, Abel M. Treatment of stiff-knee gait in cerebral palsy: a comparison by gait analysis of distal rectus femoris transfer versus proximal rectus release. J Pediatr Orthop 1990; 10: 433-441.

6 Hebert JS, Liggins AB. Gait evaluation of an automatic stance-control knee orthosis in a patient with postpoliomyelitis. Arch Phys Med Rehabil 2005; 86: 1676-1680.

7 Irby SE, Bernhardt KA, Kaufman KR. Gait of stance control orthosis users: the dynamic knee brace system. Prosthet. Orthot. Int. 2005; 29: 269-282.

8 McMillan AG, Kendrick K, Michael JW, Aronson J, Horton GW. Preliminary evidence for effectiveness of a stance control orthosis. J Prosthet Orthot 2004; 16 : 6-13.

9 Stein RB, Hayday F, Chong S, Thompson AK, Rolf R, James KB et al. Speed and efficiency in walking and wheeling with novel stimulation and bracing systems after spinal cord injury: A case study. Neuromodulation 2005; 8: 264-271.

10 Rafiaei M, Bahramizadeh M, Arazpour M, Samadian M, Hutchins SW, Farahmand $F$ et al. The gait and energy efficiency of stance control knee-ankle-foot orthoses: a literature review. Prosthet Orthot Int 2016; 40: 202-214.

11 Portnoy S, Schwartz I. Gait characteristics of post-poliomyelitis patients: Standardization of quantitative data reporting. Ann Phys Rehabil Med 2013; 56: 527-541.

12 Silver JK, Aiello DD. Polio survivors: falls and subsequent injuries. Am J Phys Med Rehabil 2002; 81: 567-570.

$13 \mathrm{Kim}$ CM, Eng JJ. Symmetry in vertical ground reaction force is accompanied by symmetry in temporal but not distance variables of gait in persons with stroke. Gait Posture 2003; 18: 23-28.

14 Kaufman KR, Irby S, Mathewson J, Wirta R, Sutherland D. Energy-efficient knee-ankle-foot orthosis: a case study. J Prosthet Orthot 1996; 8: 79.

15 Irby SE, Kaufman KR, Mathewson JW, Sutherland DH. Automatic control design for a dynamic knee-brace system. IEEE Trans Neural Syst Rehabil Eng 1999; 7: 135-139.

16 Zissimopoulos A, Fatone S, Gard SA. Biomechanical and energetic effects of a stance-control orthotic knee joint. Journal of rehabilitation research and development. Phys Med Rehab 2007; 44: 503. 